\title{
BOUNDARY VALUE PROBLEMS FOR SECOND-ORDER PARTIAL DIFFERENTIAL EQUATIONS WITH OPERATOR COEFFICIENTS
}

\author{
KUDRATILLO S. FAYAZOV AND EBERHARD SCHOCK
}

Received 9 June 2001

Let $\Omega_{T}$ be some bounded simply connected region in $\mathbb{R}^{2}$ with $\partial \Omega_{T}=\bar{\Gamma}_{1} \cap \bar{\Gamma}_{2}$. We seek a function $u(x, t),\left((x, t) \in \Omega_{T}\right)$ with values in a Hilbert space $H$ which satisfies the equation $A L u(x, t)=B u(x, t)+f\left(x, t, u, u_{t}\right),(x, t) \in \Omega_{T}$, where $A(x, t), B(x, t)$ are families of linear operators (possibly unbounded) with everywhere dense domain $D$ ( $D$ does not depend on $(x, t)$ ) in $H$ and $L u(x, t)=u_{t t}+a_{11} u_{x x}+a_{1} u_{t}+a_{2} u_{x}$. The values $u(x, t) ; \partial u(x, t) / \partial n$ are given in $\Gamma_{1}$. This problem is not in general well posed in the sense of Hadamard. We give theorems of uniqueness and stability of the solution of the above problem.

\section{Introduction}

Let $\varphi_{i}(t) \in C^{1}\left(t \geq t_{0}, i=1,2\right), t \in\left[t_{0}, T\right]$, and $\left|\varphi_{i}^{\prime}(t)\right|\left(t-t_{0}\right)^{1 / 2}<\mu(i=1,2)$, where $\mu$ is a constant. Let $\Omega_{T}$ be a bounded simply connected region in $\mathbb{R}^{2}$ defined as follows:

$$
\Omega_{T}=\left\{(x, t): 0 \leq t_{0}<t<T, \varphi_{1}(t)<x<\varphi_{2}(t), \varphi_{1}\left(t_{0}\right)=\varphi_{2}\left(t_{0}\right)\right\},
$$

and $\partial \Omega_{T}=\bar{\Gamma}_{1} \cup \bar{\Gamma}_{2}, \Gamma_{1} \cap \Gamma_{2}=\emptyset$, where

$$
\begin{aligned}
& \Gamma_{1}=\left[(x, t): x=\varphi_{i}(t)(i=1,2), t_{0} \leq t<T\right], \\
& \Gamma_{2}=\left[(x, t): t=T, \varphi_{1}(t)<x<\varphi_{2}(t)\right] .
\end{aligned}
$$

Let $D$ ( $D$ does not depend on $(x, t))$ be an everywhere dense domain in $H$, and $A(x, t), B(x, t)$ are families of linear operators (possibly unbounded) with domain $D$, let $u(x, t),\left((x, t) \in \Omega_{T}\right)$ be a function with values in the space $H$. Let $u(x, t)$ satisfy the equation

$$
A(x, t) L u(x, t)=B u(x, t)+f\left(x, t, u, u_{t}\right), \quad(x, t) \in \Omega,
$$

Copyright (C) 2001 Hindawi Publishing Corporation Abstract and Applied Analysis 6:5 (2001) 253-266 2000 Mathematics Subject Classification: 35A07 URL: http://aaa.hindawi.com/volume-6/S1085337501000628.html 
where $L u(x, t) \equiv u_{t t}+a_{11} u_{x x}+a_{2} u_{x}+a_{1} u_{t}$,

$$
a_{11} \in C^{2}\left(\Omega_{T}\right) \quad\left(a_{11}>0\right), \quad a_{1}(x, t), a_{2}(x, t) \in C^{1}\left(\bar{\Omega}_{T}\right),
$$

and in the part $\Gamma_{1}$ of the bound $\partial \Omega_{T}$ are given

$$
\left.\frac{\partial u(x, t)}{\partial n}\right|_{\Gamma_{1}}=g,\left.\quad u(x, t)\right|_{\Gamma_{1}}=f_{1}
$$

where

$$
f_{1} \in C^{1}\left(\Gamma_{1} ; H\right), \quad g \in C\left(\Gamma_{1} ; H\right) .
$$

Definition 1.1. A solution of (1.3) is called a two times smooth differentiable function which belongs to the domain of operators $A, B$ for every $(x, t) \in \Omega_{T}$ and satisfies (1.3).

The Cauchy problem is the problem to find the solution of (1.3) which satisfies condition (1.5) with $f, g \in D(A) \cap D(B)$.

The Cauchy problem (1.3), (1.4), and (1.5) is not in general well posed in the sense of Hadamard. This type of problems for differential-operator equations were studied by Krě̆n [3], Levine [5], Buchgeim [1], and others. We will prove theorems of uniqueness and stability of the solution of the Cauchy problem using Lavrent'ev's method [2, 4].

\section{Uniqueness}

Theorem 2.1. Let $A=1, L u \equiv \Delta u \equiv u_{x x}+u_{t t}$, and let $B$ be a selfadjoint constant operator. Suppose $w(x, t)$ satisfies

$$
\begin{gathered}
\triangle w=B w+f\left(t, x, w, w_{t}\right), \\
v \in C^{1}\left(\Omega_{T} ; H\right) \cap C^{2}\left(\Omega_{T} ; H\right) \cap L_{2}\left(\Omega_{T} ; D\right)
\end{gathered}
$$

is such that

$$
\triangle v=B v+f\left(t, x, v, v_{t}\right)-\varepsilon(v)
$$

is defined, and $\left.w\right|_{\Gamma_{1}}=0,\left.v\right|_{\Gamma 1}=0$. Let $u=w-v$, and

$$
\left\|f\left(t, x, v, v_{t}\right)-f\left(t, x, w, w_{t}\right)\right\|^{2} \leq c_{1} \int_{t_{0}}^{t}\|u(\tau)\|^{2} d \tau+c_{2} \int_{t_{0}}^{t}\left\|u_{\tau}(\tau)\right\|^{2} d \tau,
$$

where $c_{1}, c_{2}$ are positive constants. Then there exist constants $\Theta_{i} \geq 0, i=1,2$, such that with

$$
\gamma=\Theta_{1}\left(\max _{\Gamma_{1}}\left(\left\|u_{t}\right\|^{2}+\left\|u_{x}\right\|^{2}\right)\right)+\Theta_{2} \int_{t_{0}}^{t}\|\varepsilon(v)\|^{2} d \tau
$$


the function

$$
\Psi(t)=\ln \left(\iint_{\Omega_{t}}\|u\|^{2} d s d \tau+\gamma\right)
$$

satisfies

$$
\Psi^{\prime \prime}(t)+p \Psi^{\prime}(t)+q \geq 0
$$

for some nonnegative computable constants $p$ and $q$ which depend on $T, c_{i}(i=$ $1,2)$.

Proof. From (2.1) and (2.3) we find

$$
\triangle u=B u+\alpha\left(t, x, u, u_{t}\right)+\varepsilon(u) .
$$

We denote the estimating integral by

$$
\varphi(t)=\iint_{\Omega_{t}}\|u(s, \tau)\|^{2} d s d \tau
$$

We differentiate $\varphi(t)$, and take into account the condition $\left.u\right|_{\Gamma_{1}}=0$. We get

$$
\begin{aligned}
\varphi^{\prime}(t) & =2 \iint_{\Omega_{t}} \operatorname{Re}\left(u_{\tau}, u\right) d s d \tau \\
\varphi^{\prime \prime}(t) & =2 \iint_{\Omega_{t}} \operatorname{Re}\left(u_{\tau \tau}, u\right) d s d \tau+2 \iint_{\Omega_{t}}\left(u_{\tau}, u_{\tau}\right) d s d \tau .
\end{aligned}
$$

Taking into account (2.1) we receive

$$
\begin{aligned}
\varphi^{\prime \prime}(t)= & -2 \iint_{\Omega_{t}} \operatorname{Re}\left(u_{s s}, u\right) d s d \tau+2 \iint_{\Omega_{t}}(B u, u) d s d \tau \\
& +2 \iint_{\Omega_{t}}(\varepsilon(v), u) d s d \tau+2 \iint_{\Omega_{t}} \operatorname{Re}\left(\alpha\left(\tau, s, u, u_{\tau}\right), u\right) d s d \tau \\
& +2 \iint_{\Omega_{t}}\left(u_{\tau}, u_{\tau}\right) d s d \tau
\end{aligned}
$$

where $\alpha\left(t, s, u, u_{t}\right)=f\left(t, s, w, w_{t}\right)-f\left(t, s, v, v_{t}\right)$.

Using the integration by part and taking into account the condition $\left.u\right|_{\Gamma_{1}}=0$, (2.8) we get from (2.11)

$$
\begin{aligned}
\varphi^{\prime \prime}(t)= & -2 \iint_{\Omega_{t}}\left[\left(u_{s}, u_{s}\right)+\left(u_{\tau}, u_{\tau}\right)+(B u, u)\right] d s d \tau \\
& +2 \iint_{\Omega_{t}} \operatorname{Re}\left(\alpha\left(\tau, s, u, u_{\tau}\right)+\varepsilon(v), u\right) d s d \tau .
\end{aligned}
$$


We differentiate

$$
p(t)=\int_{\varphi_{1}(t)}^{\varphi_{2}(t)}\left\|u_{s}(s, t)\right\|^{2} d s
$$

and obtain

$$
\begin{aligned}
p^{\prime}(t)= & \int_{\varphi_{1}(t)}^{\varphi_{2}(t)} \frac{\partial}{\partial t}\left(u_{s}, u_{s}\right) d s+\sum_{i=1}^{2}(-1)^{i} \varphi_{i}^{\prime}(t)\left\|u_{s}\left(\varphi_{i}(t), t\right)\right\|^{2} \\
= & -\int_{\varphi_{1}(t)}^{\varphi_{2}(t)} 2 \operatorname{Re}\left(u_{t}, u_{s s}\right) d s+2 \sum_{i=1}^{2}(-1)^{i} \operatorname{Re}\left(u_{t}\left(\varphi_{i}(t), t\right) u_{s}\left(\varphi_{i}(t), t\right)\right) \\
& +\sum_{i=1}^{2}(-1)^{i} \varphi_{i}^{\prime}(t)\left\|u_{s}\left(\varphi_{i}(t), t\right)\right\|^{2} \\
= & \frac{d}{d t} \int_{\varphi_{1}(t)}^{\varphi_{2}(t)}\left[\left(u_{t}, u_{t}\right)+(B u, u)\right] d s-2 \int_{\varphi_{1}(t)}^{\varphi_{2}(t)} \operatorname{Re}\left(\alpha\left(t, s, u, u_{t}\right)+\varepsilon(v), u_{t}\right) d s \\
& +\sum_{i=1}^{2}(-1)^{i}\left\{\varphi_{i}^{\prime}(t)\left[\left\|u_{s}\left(\varphi_{i}(t), t\right)\right\|^{2}-\left\|u_{t}\left(\varphi_{i}(t), t\right)\right\|^{2}\right]\right. \\
& \left.+2 \operatorname{Re}\left(u_{t}\left(\varphi_{i}(t), t\right), u_{s}\left(\varphi_{i}(t), t\right)\right)\right\} .
\end{aligned}
$$

By deducing this formula we use (2.8) and integration by parts. Integrating (2.14) from $t_{0}$ till $t$ we get the following:

$$
\begin{aligned}
\int_{\varphi_{1}(t)}^{\varphi_{2}(t)} & \left(\left\|u_{s}\right\|^{2}+(B u, u)\right) d s \\
= & \int_{\varphi_{1}(t)}^{\varphi_{2}(t)}\left\|u_{t}\right\|^{2} d s-2 \iint_{\Omega_{t}} \operatorname{Re}\left(\alpha\left(\tau, s, u, u_{\tau}\right)+\varepsilon(v), u_{\tau}\right) d s d \tau+b_{0}(t),
\end{aligned}
$$

where

$$
\begin{array}{r}
b_{0}(t)=\sum_{i=1}^{2}(-1)^{i} \int_{t_{0}}^{t} \varphi_{i}^{\prime}(\tau)\left[\left\|u_{s}\left(\varphi_{i}(t), t\right)\right\|^{2}-\left\|u_{t}\left(\varphi_{i}(\tau), \tau\right)\right\|^{2}\right] \\
+2 \operatorname{Re}\left(u_{\tau}\left(\varphi_{i}(\tau), \tau\right), u_{s}\left(\varphi_{i}(\tau), \tau\right)\right) d \tau .
\end{array}
$$

Substituting (2.15) in (2.12) we get

$$
\begin{aligned}
\varphi^{\prime \prime}(t)= & 4 \iint_{\Omega_{t}}\left\|u_{\tau}\right\|^{2} d s d \tau-4 \int_{t_{0}}^{t} \iint_{\Omega_{t}} \operatorname{Re}\left(\alpha+\varepsilon(v), u_{\tau}\right) d s d \tau_{1} d \tau \\
& +2 \iint_{\Omega_{t}} \operatorname{Re}(\alpha+\varepsilon(v), u) d s d \tau+\int_{t_{0}}^{t} b_{0}(\tau) d \tau .
\end{aligned}
$$


We notice that

$$
\left|\int_{t_{0}}^{t} b_{0}(\tau) d t\right| \leq \Theta_{0} \sum_{i=1}^{2} \int_{t_{0}}^{t}(t-\tau)\left|\varphi_{i}^{\prime}(\tau)\right|\left[\left\|u_{\tau}\right\|^{2}+\left\|u_{s}\right\|^{2}\right]_{s=\varphi_{i}(t)} d \tau \leq \gamma
$$

where $\gamma$ is defined by (2.3). From (2.17) we can write

$$
\begin{aligned}
& 4 \int_{t_{0}}^{t} \iint_{\Omega_{t}}\left\|u_{\tau_{1}}\left(\tau_{1}, s\right)\right\|^{2} d s d \tau_{1} d \tau \\
& \leq \varphi^{\prime}(t)+4\left|\int_{t_{0}}^{t} \iint_{\Omega_{t}}\left(\alpha+\varepsilon(v), u_{\tau_{2}}\right) d s d \tau_{2} d \tau_{1} d \tau\right| \\
& +2\left|\int_{t_{0}}^{t} \iint_{\Omega_{t}} \operatorname{Re}(\alpha+\varepsilon(v), u) d s d \tau_{1} d \tau\right|+\left|\int_{t_{0}}^{t} \int_{t_{0}}^{\tau} b_{0}\left(\tau_{1}\right) d \tau_{1} d \tau\right|
\end{aligned}
$$

Using the Cauchy and Bellman inequalities and the inequality

$$
|a b| \leq \frac{a^{2}}{2} \beta+\frac{b^{2}}{2 \beta}, \quad \beta>0
$$

from (2.19) we receive the following inequality:

$$
\begin{aligned}
& \int_{t_{0}}^{t} \iint_{\Omega_{t}}\left\|u_{\tau_{1}}\left(\tau_{1}, s\right)\right\|^{2} d s d \tau_{1} d \tau \\
& \quad \leq k_{1} \varphi^{\prime}(t)+k_{2} \varphi(t)+k_{3} \int_{t_{0}}^{t} \iint_{\Omega_{t}}\|\varepsilon(v)\|^{2} d s d \tau_{1} d \tau+T \gamma
\end{aligned}
$$

where $k_{1}, k_{2}$, and $k_{3}$ are nonnegative constants which depend on $T$ and the constants $c_{1}$ and $c_{2}$. Then for $\varphi^{\prime \prime}(t)$ we get the following:

$$
\begin{aligned}
\varphi^{\prime \prime}(t) \geq 4 & \iint_{\Omega_{t}}\left\|u_{\tau}(s, \tau)\right\|^{2} d s d \tau \\
& \quad-k_{4} \varphi^{\prime}(t)-k_{5} \varphi(t)-k_{6} \iint_{\Omega_{t}}\|\varepsilon(v)\|^{2} d \tau d s-k_{7} \gamma .
\end{aligned}
$$

We consider now the function $\psi(t)=\ln [\varphi(t)+\gamma]$, using the Cauchy inequality,

$$
|a b| \leq \frac{a^{2}}{2} \beta+\frac{b^{2}}{2 \beta}, \quad \beta>0
$$


and (2.22) we transform the second derivative

$$
\begin{gathered}
\psi^{\prime \prime}(t)=\frac{\varphi^{\prime \prime}(t) \cdot(\varphi(t)+\gamma)-\left(\varphi^{\prime}(t)\right)^{2}}{(\varphi(t)+\gamma)^{2}} \\
\geq \frac{1}{(\varphi(t)+\gamma)^{2}}\left\{\left(4 \iint_{\Omega_{t}}\left\|u_{\tau}(s, \tau)\right\|^{2} d s d \tau-k_{4} \varphi^{\prime}(t)\right.\right. \\
\left.\quad-k_{5} \varphi(t)-k_{6} \iint_{\Omega_{t}}\|\varepsilon(u)\|^{2} d s d \tau-k_{7} \gamma\right) \\
\left.\quad \times\left(\iint_{\Omega_{t}}\|u\|^{2} d s d \tau+\gamma\right)-4\left(\iint_{\Omega t} \operatorname{Re}\left(u_{\tau}, u\right) d s d \tau\right)^{2}\right\} \\
\geq-k_{8} \frac{\varphi^{\prime}}{\varphi(t)+\gamma}-k_{9},
\end{gathered}
$$

or

$$
\psi^{\prime \prime}+p \psi^{\prime}+q \geq 0
$$

where $p, q$ are nonnegative constants that depend on $T$ and the constants $c_{1}, c_{2}$. Theorem 2.1 is proved.

Remark 2.2. It is known from the theory of ordinary differential equations if the function $\psi(t)$ satisfies the inequality (2.7), then it satisfies the following inequality:

$$
\psi(t) \leq \psi_{0}(t),
$$

where $\psi_{0}(t)$ is a solution of the differential equation

$$
\psi_{0}^{\prime \prime}(t)+p \psi_{0}^{\prime}(t)+q=0
$$

with boundary conditions $\psi_{0}\left(t_{0}\right)=\psi\left(t_{0}\right), \psi_{0}(T)=\psi(T)$. It is not difficult to see that

$$
\begin{gathered}
\psi_{0}(t)=\ell_{1}+\ell_{2} \exp (-p t)-q \frac{t}{p} \\
\ell_{1}=\frac{\psi(T) \exp \left(-p t_{0}\right)-\psi\left(t_{0}\right) \exp (-p T)+(q / p)\left(T \exp \left(-p t_{0}\right)-t_{0} \exp (-p T)\right)}{\exp \left(-p t_{0}\right)-\exp (-p T)} \\
\ell_{2}=\psi(0)-\psi(T)+\frac{q}{p}\left(t_{0}-T\right)\left(\exp \left(-p t_{0}\right)-\exp (-p T)\right) \\
\psi(t) \leq(1-\omega(t)) \psi\left(t_{0}\right)+\omega(t) \psi(T)+q \frac{\omega(t)\left(T-t_{0}\right)+\left(t_{0}-t\right)}{p}
\end{gathered}
$$


where

$$
\omega(t)=\frac{\exp \left(-p t_{0}\right)-\exp (-p t)}{\exp \left(-p t_{0}\right)-\exp (-p T)} .
$$

Further, it is not difficult to see, from (2.7), that

$$
\iint_{\Omega_{t}}\|u\|^{2} d s d \tau \leq c(t) \gamma^{1-\omega(t)}\left\{\iint_{\Omega_{t}}\|u\|^{2} d s d \tau+\gamma\right\}^{\omega(t)}-\gamma .
$$

COROLlary 2.3. The solution of the Cauchy problem for (1.3) is unique in the space

$$
C^{1}\left(\bar{\Omega}_{T} ; H\right) \cap C^{2}\left(\Omega_{T} ; H\right) \cap L_{2}\left(\Omega_{T} ; D\right) .
$$

Proof. Let $v, w$ be solutions of the Cauchy problem for (2.1) and (2.3), respectively. Then $u=w-v$ is the solution of the homogeneous Cauchy problem for the equation

$$
\triangle u=B u+\alpha\left(t, u, u_{t}\right)
$$

and $\gamma=0$ and from (2.30) follows $u \equiv 0$ or $w \equiv v$. Corollary 2.3 is proved.

From inequality (2.30) it is easy to see that the following corollary follows.

Corollary 2.4. The solution of the Cauchy problem for (1.3) is stable in the space

$$
\begin{gathered}
C^{1}\left(\bar{\Omega}_{T} ; H\right) \cap C^{2}\left(\Omega_{T} ; H\right) \cap L_{2}\left(\Omega_{T} ; D\right), \\
u \in\left\{u: \iint_{\Omega_{t}}\|u\|^{2} d s d \tau \leq M\right\} .
\end{gathered}
$$

\section{Stability}

Let $A$ be a constant selfadjoint operator and $(A u, u)>0$ for all $u \neq 0,(A u, u)=$ 0 if and only if $u=0$. Let $B(x, t)$ be a selfadjoint operator for every $(x, y) \in D$ and with

$$
\begin{aligned}
c=\max \left[\max _{(x, t) \in\left[\Omega_{T}\right]} \frac{\left(\left|a_{11_{r}}\right|+\left|a_{1} / 2\right|+\left|a_{11_{s}} / 2\right|\right)}{a_{11}}\right. & \\
& \left.\max \left(\left|\frac{a_{11_{s}}}{2}\right|+\left|\frac{\left(a_{1}\right)}{2}\right|+\left|\left(a_{2}\right)\right|+2 c_{1}+\beta_{9}\right)\right]
\end{aligned}
$$

satisfies

$$
\left(B_{t} u, u\right) \geq-c(B u, u) .
$$

Let $w$ satisfy

$$
A L w=B w+F\left(x, t, w, w_{t}\right)
$$


and $v$ satisfy

$$
A L v=B v+f\left(x, t, v, v_{t}\right)-\varepsilon(v) .
$$

Let $u=w-v$ and $\alpha=f\left(x, t, w, w_{t}\right)-f\left(x, t, v, v_{t}\right)$ and

$$
\|\alpha\|^{2} \leq c_{1}\|u\|^{2}+c_{2}\left\|u_{t}\right\|^{2}
$$

then

$$
A L u=B u+\alpha\left(x, t, u, u_{t}\right)+\varepsilon(v),
$$

where $c_{1}, c_{2}$ are constants.

THEOREM 3.1. Let the coefficients $a_{11}, a_{1}$, and $a_{2}$ satisfy condition (1.4). If the solution of (3.6) is equal to zero on $\Gamma_{1}$ and satisfies the inequality

$$
\begin{gathered}
\iint_{\Omega_{T}}(u, A u) d s d \tau \leq M, \\
\gamma=\Theta \max _{\Gamma_{1}}\left[\left(u_{t}, A u_{t}\right)+\left(u_{x}, A u_{x}\right)\right]+\iint_{\Omega_{T}}\|\varepsilon(v)\|^{2} d s d \tau,
\end{gathered}
$$

then for $u \in C^{1}\left(\bar{\Omega}_{T} ; H\right) \cap C^{2}\left(\Omega_{T} ; H\right) \cap L_{2}\left(\Omega_{T} ; D\right)$ the following inequality is true

$$
\iint_{\Omega_{t}}(u, A u) d s d \tau \leq \gamma^{1-\omega(t)}(M+\gamma)^{\omega(t)} c(t)-\gamma
$$

where

$$
\begin{aligned}
c(t) & =\exp \frac{q(\gamma(t) T-t)}{p}, \\
\omega(t) & =\frac{\exp \left(-p t_{0}\right)-\exp (-p t)}{\exp \left(-p t_{0}\right)-\exp (-p T)}
\end{aligned}
$$

and $\Theta, p, q$ are constants that depend on the coefficients $T$ and $c_{i}, i=1,2$.

Proof. Let

$$
F(t)=\iint_{\Omega_{t}}(u, A u) d s d \tau
$$

then

$$
\begin{aligned}
F^{\prime}(t) & =\iint_{\Omega_{t}} 2 \operatorname{Re}\left(u_{\tau}, A u\right) d s d \tau \\
F^{\prime \prime}(t) & =\iint_{\Omega_{t}} 2 \operatorname{Re}\left(u_{\tau \tau}, A u\right) d s d \tau+F(t) \\
& =\iint_{\Omega_{t}} 2\left(u_{\tau}, A u_{\tau}\right) d s d \tau
\end{aligned}
$$


because $\left.u\right|_{\Gamma_{1}}=0$. We transform the first term in the expression for $F^{\prime \prime}(t)$ using (3.6) and integration by parts

$$
\begin{aligned}
\iint_{\Omega_{t}} 2 & \operatorname{Re}\left(u_{\tau \tau}, A u\right) d s d \tau \\
= & \iint_{\Omega_{t}} 2(B u, u) d s d \tau \iint_{\Omega_{t}} 2 a_{11}\left(u_{s}, A u_{s}\right) d s d \tau \\
& +\iint_{\Omega_{t}} 2 \operatorname{Re}\left(u, \alpha\left(s, \tau, u, u_{\tau}\right)\right) d s d \tau+\iint_{\Omega_{t}} 2 \operatorname{Re}(u, \varepsilon(v)) d s d \tau \\
& -\iint_{\Omega_{t}} 2 \operatorname{Re}\left(-a_{11_{s}}+a_{2}\right)\left(u_{s}, A u\right) d s d \tau-\iint_{\Omega_{t}} 2 a_{1} \operatorname{Re}\left(u_{\tau}, A u\right) d s d \tau .
\end{aligned}
$$

After the transform we get

$$
\begin{gathered}
F^{\prime \prime}(t)=\iint_{\Omega_{1}} 2\left(\left(u_{\tau}, A u_{\tau}\right)+a_{11}\left(u_{s}, A u_{s}\right)+(u, B u)\right) d s d \tau \\
+\iint_{\Omega_{1}} 2\left[\left(a_{11_{s}}-a_{2}\right)\left(u_{s}, A u\right)+\operatorname{Re}\left(u, \alpha\left(s, \tau, u, u_{\tau}\right)\right)\right. \\
\left.-\operatorname{Re}(u, \varepsilon(v))-a_{1} \operatorname{Re}\left(u_{\tau}, A u\right)\right] d s d \tau
\end{gathered}
$$

From here and from (1.4) using the conditions for the coefficients and Hölder's inequality, we get

$$
\begin{aligned}
F^{\prime \prime}(t) \geq & \iint_{\Omega t} 2\left(\left(u_{\tau}, A u_{\tau}\right)+a_{11}\left(u_{s}, A u_{s}\right)+(u, B u)\right) d s d \tau \\
& -2 \beta_{1}\left[\iint_{\Omega t}\left(u_{s}, A u_{s}\right) d s d \tau F(t)\right]^{1 / 2} \\
& -2 \beta_{2}\left[\iint_{\Omega t}\left(u_{\tau}, A u_{\tau}\right) d s d \tau F(t)\right]^{1 / 2} \\
& -2 \beta_{3} F(t)-\beta_{4}\left[\iint_{\Omega_{1}}\left(u_{\tau}, A u_{\tau}\right) d s d \tau \iint_{\Omega_{1}}(u, A u) d s d \tau\right]^{1 / 2} \\
& -\beta_{5}\left[\iint_{\Omega_{t}}\|\varepsilon(v)\| d s d \tau \iint_{\Omega_{t}}(u, A u) d s d \tau\right]^{1 / 2} \\
\geq & \iint_{\Omega_{t}} 2\left(\left(u_{\tau}, A u_{\tau}\right)+a_{11}\left(u_{s}, A u_{s}\right)+(u, B u)\right) d s d \tau \\
& -\left(\frac{\beta_{1}^{2}}{a_{11}}+\left(\frac{\left(2 \beta_{2}+\beta_{4}\right)}{2}\right)^{2}-2 \beta_{3}+\frac{\beta_{5}^{2}}{2}\right) F(t) \\
& -0.5 \iint_{\Omega_{t}}\|\varepsilon(v)\|^{2} d s d \tau .
\end{aligned}
$$


From the last inequality it follows that

$$
\begin{gathered}
\int_{t_{0}}^{t} \iint_{\Omega_{t}}\left(\left(u_{\tau_{1}}, A u_{\tau_{1}}\right)+a_{11}\left(u_{s}, A u_{s}\right)+(u, B u)\right) d s d \tau_{1} d \tau \\
\leq F^{\prime}(t)+\beta_{6} F(t)+0.5 \iint_{\Omega_{t}}\|\varepsilon(v)\|^{2} d s d \tau .
\end{gathered}
$$

Differentiating the first integral on the right-hand side of (3.12), we get

$$
\begin{aligned}
& \frac{d}{d t} \iint_{\Omega_{t}} a_{11}\left(u_{s}, A u_{s}\right) d s d \tau \\
& =\frac{d}{d t} \iint_{\Omega_{t}}\left\{\left(u_{\tau}, A u_{\tau}\right)-(B u, u)\right\} d s d \tau \\
& \quad+\int_{t_{0}}^{t} \sum_{i=1}^{2}\left\{( - 1 ) ^ { i } \varphi _ { i } ^ { \prime } ( t ) \left[a_{11}\left(u_{s}\left(\varphi_{i}(\tau), \tau\right), A u_{s}\left(\varphi_{i}(\tau), \tau\right)\right)\right.\right. \\
& \left.\quad-\left(u_{\tau}\left(\varphi_{i}(\tau), \tau\right), A u_{\tau}\left(\varphi_{i}(\tau), \tau\right)\right)\right] \\
& \left.\quad+a_{11}\left(u_{\tau}\left(\varphi_{i}(\tau), \tau\right), A u_{s}\left(\varphi_{i}(\tau), \tau\right)\right)\right\} d \tau \\
& \quad+\iint_{\Omega_{t}}\left[a_{11_{\tau}}\left(u_{s}, A u_{s}\right)-a_{11_{s}}\left(u_{\tau}, A u_{s}\right)\right] d s d \tau \\
& \quad+\iint_{\Omega_{t}}\left[\left(u, B u_{\tau}\right)+a_{2}\left(u_{\tau}, A u_{\tau}\right)+a_{1}\left(u_{\tau}, A u_{s}\right)\right. \\
& \left.\quad-\left(u_{\tau}, \alpha+\varepsilon(v)\right)\right] d s d \tau .
\end{aligned}
$$

After this transformation $F^{\prime \prime}(t)$ takes the form

$$
\begin{aligned}
F^{\prime \prime}(t)= & 4 \iint_{\Omega_{t}}\left(u_{\tau}, A u_{\tau}\right) d s d \tau \\
& +2 \int_{t_{0}}^{t} \iint_{\Omega_{t}}\left[a_{11_{\tau}}\left(u_{s}, A u_{s}\right)-a_{11_{s}} \operatorname{Re}\left(u_{\tau}, A u_{s}\right)\right. \\
& \left.\quad+\operatorname{Re}\left(u, B u_{\tau}\right)+a_{2}\left(u_{\tau}, A u_{\tau}\right)+a_{1} \operatorname{Re}\left(u_{\tau}, A u_{s}\right)\right] d s d \tau \\
& +2 \int_{t_{0}}^{t} \iint_{\Omega_{t}} \operatorname{Re}\left(u_{\tau}, \alpha+\varepsilon(v)\right) d s d \tau \\
& +\iint_{\Omega t}\left\{\left(a_{11_{s}}-a_{2}\right) \operatorname{Re}\left(u_{s}, A u\right)+2 \operatorname{Re}\left(u_{\tau}, \alpha+\varepsilon(v)\right)\right. \\
& \left.\quad-2 a_{1} \operatorname{Re}\left(u_{\tau}, A u_{\tau}\right)\right\} d s d \tau+b_{0}(\tau)
\end{aligned}
$$


where

$$
\begin{array}{r}
b_{0}(\tau)=\int_{t_{0}}^{t}(t-\tau) \sum_{i=1}^{2}\left\{( - 1 ) ^ { i } \varphi _ { i } ^ { \prime } ( t ) \left[a_{11}\left(u_{x}\left(\varphi_{i}(\tau), \tau\right), A u_{x}\left(\varphi_{i}(\tau), \tau\right)\right)\right.\right. \\
\left.-\left(u_{\tau}\left(\varphi_{i}(\tau), \tau\right), A u_{\tau}\left(\varphi_{i}(\tau), \tau\right)\right)\right] \\
\left.+a_{11} \operatorname{Re}\left(u_{\tau}\left(\varphi_{i}(\tau), \tau\right), A u_{x}\left(\varphi_{i}(\tau), \tau\right)\right)\right\} d \tau
\end{array}
$$

We remark that

$$
\begin{gathered}
\left|b_{0}(\tau)\right| \leq \sum_{i=1}^{2} \int_{t_{0}}^{t}(t-\tau)\left\{a _ { 1 1 } \left[\left|\varphi_{i}^{\prime}\right|\left(u_{x}\left(\varphi_{i}(\tau), \tau\right), A u_{x}\left(\varphi_{i}(\tau), \tau\right)\right)\right.\right. \\
\left.+\left|2 \operatorname{Re}\left(u_{x}\left(\varphi_{i}(\tau), \tau\right), A u_{\tau}\left(\varphi_{i}(\tau), \tau\right)\right)\right|\right] \\
\left.+\left|\varphi_{i}^{\prime}\right|\left(u_{\tau}\left(\varphi_{i}(\tau), \tau\right), A u_{\tau}\left(\varphi_{i}(\tau), \tau\right)\right)\right\} d \tau
\end{gathered}
$$$$
\leq \gamma_{1} \text {. }
$$

We denote

$$
s^{2}=\iint_{\Omega_{t}}\left(u_{\tau}, A u_{\tau}\right) d s d \tau \iint_{\Omega_{t}}(u, A u) d s d \tau-\left(\iint_{\Omega_{t}}\left(u_{\tau}, A u_{\tau}\right) d s d \tau\right)^{2}
$$

Then using Hölder's inequality, (1.4), (3.15) we obtain from (3.17)

$$
\begin{aligned}
& F^{\prime \prime}(t) \geq 4 \iint_{\Omega_{t}}\left(u_{\tau}, A u_{\tau}\right) d s d \tau \\
& -2 \int_{t_{0}}^{t} \iint_{\Omega_{t}}\left\{\left[\left|a_{11_{r}}\right|+\frac{\left|a_{1_{1}}\right|}{2}+\frac{\left|a_{11_{s}}\right|}{2}\right]\left(u_{s}, A u_{s}\right)\right. \\
& +\left[\frac{\left|a_{11_{s}}\right|}{2}+\frac{\left|a_{1}\right|}{2}+\frac{\left|a_{2}\right|}{2}\right]\left(u_{\tau}, A u_{\tau}\right) \\
& \left.+\left|\operatorname{Re}\left(u, B_{\tau} u\right)\right|\right\} d s d \tau d \tau_{1} \\
& -2 c_{2} \int_{t_{0}}^{t} \iint_{\Omega_{\tau_{1}}}\left(u_{\tau_{1}}, A u_{\tau_{1}}\right) d s d \tau d \tau_{1} \\
& -2 c_{1}\left\{\int_{t_{0}}^{t} \iint_{\Omega_{\tau_{1}}}\left(u_{\tau_{1}}, A u_{\tau_{1}}\right) d s d \tau d \tau_{1} F(t)\right\}^{1 / 2} \\
& -2\left\{\int_{t_{0}}^{t} \iint_{\Omega_{\tau_{1}}}\left(u_{\tau_{1}}, A u_{\tau_{1}}\right) d s d \tau d \tau_{1} \int_{t_{0}}^{t} \iint_{\Omega_{t}}\|\varepsilon(v)\|^{2} d s d \tau d \tau_{1}\right\}^{1 / 2}
\end{aligned}
$$


BVP with operator coefficients

$$
\begin{aligned}
& -\iint_{\Omega_{t}}\left|a_{11_{s}}-a_{2}\right| \operatorname{Re}(u, A u) d s d \tau-2 c_{1} \iint_{\Omega_{t}} \operatorname{Re}(u, A u) d s d \tau \\
& -c_{2}\left\{\iint_{\Omega_{t}} \operatorname{Re}\left(u_{\tau}, A u_{\tau}\right) d s d t\right\}^{1 / 2} \\
& -2 \beta\left\{\iint_{\Omega_{t}} \operatorname{Re}\left(u_{\tau}, A u_{\tau}\right) d s d \tau F(t)\right\}^{1 / 2}-\gamma \\
\geq & 4 \iint_{\Omega_{t}}\left(u_{\tau}, A u_{\tau}\right) d s d \tau \\
& -\int_{t_{0}}^{t} \iint_{\Omega_{t}}\left\{c a_{11}\left(u_{s}, A u_{s}\right)+c\left(u_{\tau}, A u_{\tau} a u\right)+c(u, B u)\right\} d s d \tau d \tau_{1} \\
& -\beta_{6} F^{\prime}(t)-\beta_{7} F(t)-\beta_{8} s-\gamma \\
\geq & 4 \iint_{\Omega_{t}}\left(u_{\tau}, A u_{\tau}\right) d s d \tau-\beta_{6} F^{\prime}(t)-\beta_{7} F(t)-\beta_{s}-\gamma, \\
(c= & \left.\max \left\{\frac{2\left|a_{11_{r}}\right|+\left|a_{1}\right|+\left|a_{11_{s}}\right|}{2\left|a_{11}\right|},\left[\frac{\left|a_{11_{s}}\right|}{2}+\frac{\left|a_{1}\right|}{2}+\left|a_{2}\right|+2 c_{1}+\beta_{9}\right]\right\}\right) .
\end{aligned}
$$

Let $\phi(t)=\ln [F(t)+\gamma]$, then

$$
\begin{aligned}
\Phi^{\prime \prime}(t)= & \frac{\left(F^{\prime \prime}(t)(F(t)+\gamma)-\left(F^{\prime}(t)\right)^{2}\right)}{(F(t)+\gamma)^{2}} \\
\geq & \frac{4 \iint_{\Omega_{t}}\left(u_{\tau}, A u_{\tau}\right) d s d \tau \iint_{\Omega_{t}}(u, A u) d s d \tau-\left(2 \iint_{\Omega_{t}}\left(u_{\tau}, A u\right) d s d \tau\right)^{2}}{(F(t)+\gamma)^{2}} \\
& +4 \frac{\iint_{\Omega t}\left(u_{\tau}, A u_{\tau}\right) d s d \tau \gamma}{(F(t)+\gamma)^{2}}-\frac{\beta_{9} F^{\prime}(t)+\beta_{10} F(t)+\beta_{11} S+\gamma}{F(t)+\gamma} \\
\geq & -p \frac{F^{\prime}(t)}{F(t)+\gamma}-q .
\end{aligned}
$$

Inequality (3.8) follows from (3.22), and the theorem is proved.

Remark 3.2. We can obtain similar results for arbitrary second order elliptic operators $L$, using the methods of [4].

Remark 3.3. For

$$
B(x, t)=B_{1}(x, t)+i B_{2}(x, t),
$$

where $B_{1}(x, t)$ and $B_{2}(x, t)$ are selfadjoint operators for all $(x, t) \in \Omega_{T}$ and $\lambda$, $\mu, c$ are constants such that $(A u, u) \geq \lambda(u, u)>0,\left\|B_{2} u\right\|^{2} \leq \mu(A u, u)$, and $-\left(B_{1}\right)_{t} \leq c B_{t}$, a similar result is valid. 
Example 3.4. We consider the equation

$$
\operatorname{sgn}(y)\left(u_{t t}(t, x, y)+u_{x x}(t, x, y)\right)=u_{y y}(t, x, y)
$$

in the region $Q=(-1,1) \times \Omega_{T}\left(\Omega_{T}\right.$ is defined as above). This equation is a mixed type equation. We will consider the problem of finding the solution of this equation in $Q(y \neq 0)$ which satisfies the following boundary conditions:

$$
\left.u(t, x, y)\right|_{\Gamma_{i}^{\prime}}=f,\left.\quad \frac{\partial u(t, x, y)}{\partial n}\right|_{\Gamma_{1}^{\prime}}=g,
$$

where $\Gamma_{1}^{\prime}=\Gamma_{1} \times(-1,1), \Gamma_{2}^{\prime}=\Gamma_{2} \times(-1,1)$;

$$
\begin{gathered}
u(t, x,-1)=0, \quad u(t, x, 1)=0, \quad(t, x) \in \Omega_{T} ; \\
u(t, x,-0)=u(t, x,+0), \\
\frac{\partial u(t, x,-0)}{\partial y}=\frac{\partial u(t, x,+0)}{\partial y} .
\end{gathered}
$$

Here $B$ is a selfadjoint positive definite in $L_{2}(-1,1)$ operator which is generated by the differential expression

$$
B u=-\frac{\partial^{2} u}{\partial y^{2}}
$$

and with boundary conditions $\left.u\right|_{y=-1}=\left.u\right|_{y=1}=0$. We define the operator $A$ as the operator of multiplication with the function $\operatorname{sgn}(y)$. This problem is an ill-posed problem in the sense of Hadamard, since continuous dependence of the solution from the data is absent in it.

Using the above described method we can prove the following result.

THEOREM 3.5. If a solution of this problem becomes zero on the surface $\Gamma_{1}^{\prime}$ and satisfies

$$
\begin{gathered}
\int_{0}^{T} \int_{\varphi_{1}(t)}^{\varphi_{2}(t)} \int_{-1}^{1}\left(u_{y}(t, x, y)\right)^{2} d y d s d t \leq M \\
\Theta \max _{\Gamma_{1}}\left(\int_{-1}^{1}\left(u_{y x}\right)^{2} d y+\int_{-1}^{1}\left(u_{y t}\right)^{2} d y+\int_{-1}^{1}\left|\operatorname{sign}(x)\left(u_{y y}\right)^{2} d y\right|\right)=\gamma,
\end{gathered}
$$

( $\Theta$ is constant that depends on $\left.T, \Gamma_{1}\right)$, then the inequality

$$
\int_{0}^{t} \int_{\varphi_{1}(t)}^{\varphi_{2}(t)} \int_{-1}^{1}\left(u_{y}(t, x, y)\right)^{2} d y d s d t \leq \gamma^{1-\omega_{1}(t)}(M+\gamma)^{\omega_{1}(t)} c_{1}(t)-\gamma
$$

is valid, where $c_{1}(t)=\exp (t(T-t) / 2), \omega_{1}(t)=(1-t / T)$.

From this theorem one can easily see that the uniqueness and the conditional stability of the solution of this problem follows. 


\section{References}

[1] A. L. Buchgeim, Ill-posed problems of the numerical theory and tomography, Siberian Math. J. 33 (1992), 27-41.

[2] K. S. Fayazov and M. M. Lavrent'ev, Cauchy problem for partial differential equations with operator coefficients in space, J. Inverse Ill-Posed Probl. 2 (1994), no. 4, 283-296. MR 95k:35215. Zbl 0820.35133.

[3] S. G. Kreı̆n, Lineikhye Differentsialnye Uravneniya V Banakhovom Prostranstve. [Linear Differential Equations in a Banach Space], Izdat. "Nauka", Moscow, 1967 (Russian). MR 40\#508.

[4] M. M. Lavrent'ev, On the problem of Cauchy for linear elliptic equations of the second order, Dokl. Akad. Nauk SSSR (N. S.) 112 (1957), 195-197 (Russian). MR 19,553i. Zbl 077.30001.

[5] H. A. Levine, Logarithmic convexity and the Cauchy problem for some abstract second order differential inequalities, J. Differential Equations 8 (1970), 34-55. MR 41\#3945. Zbl 194.13101.

Kudratillo S. Fayazov: Department of Mathematics, National University of Usbekistan, TashKent, 700090 TashKent, VuZgorodok, UsBekistan

E-mail address: k.fayazov@gmx.co.uk

Eberhard Schock: Department of Mathematics, University of Kaiserslautern, P.O. Box 3049, 67653 KaisERSLAUTERn, GERMANY

E-mail address: schock@mathematik.uni-kl.de 


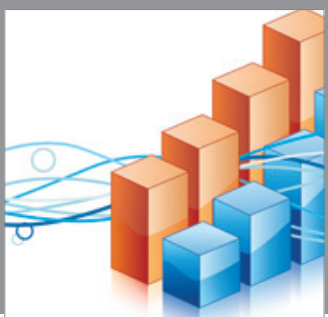

Advances in

Operations Research

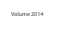

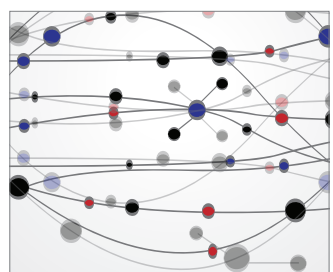

\section{The Scientific} World Journal
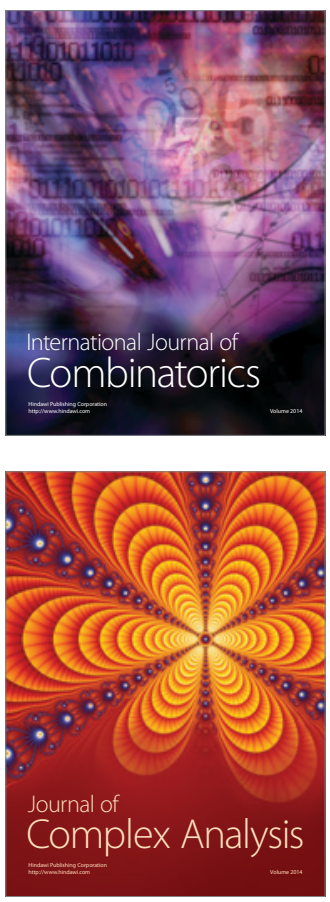

International Journal of

Mathematics and

Mathematical

Sciences
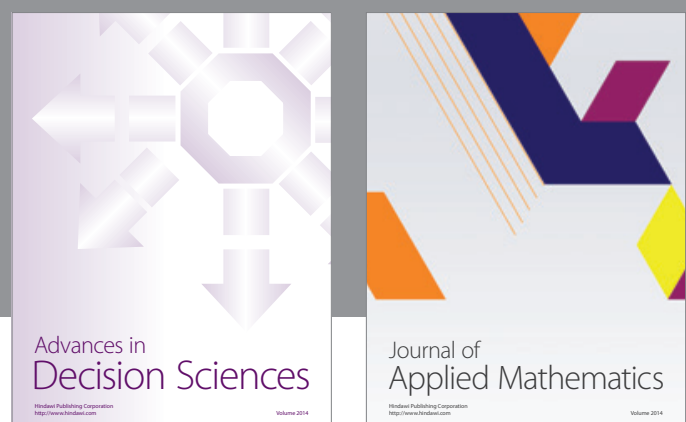

Journal of

Applied Mathematics
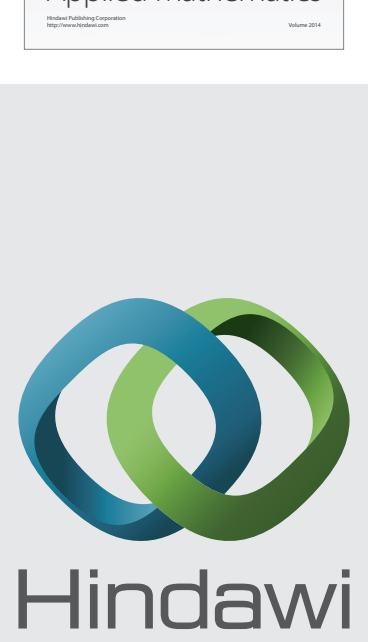

Submit your manuscripts at http://www.hindawi.com
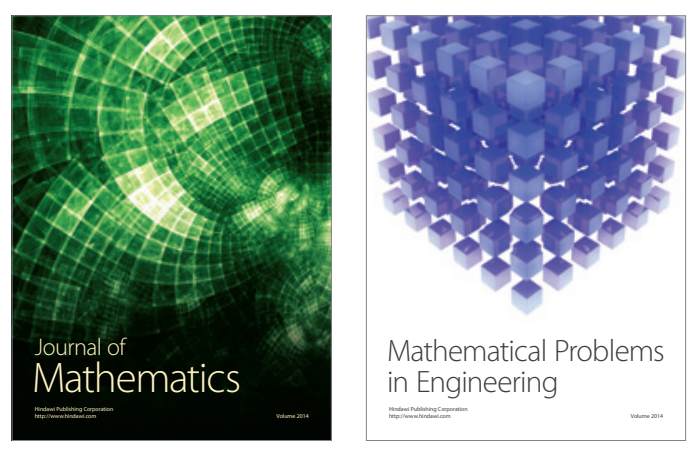

Mathematical Problems in Engineering
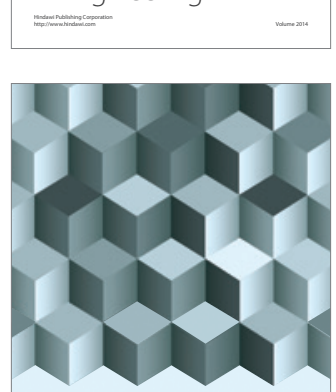

Journal of

Function Spaces
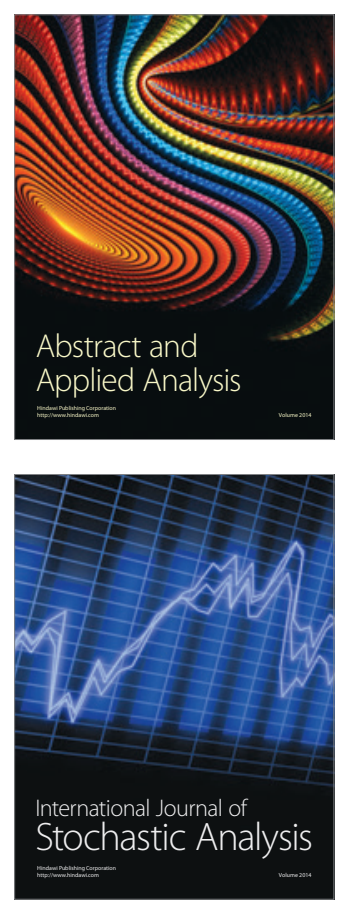

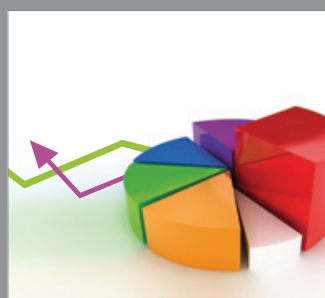

ournal of

Probability and Statistics

Promensencen
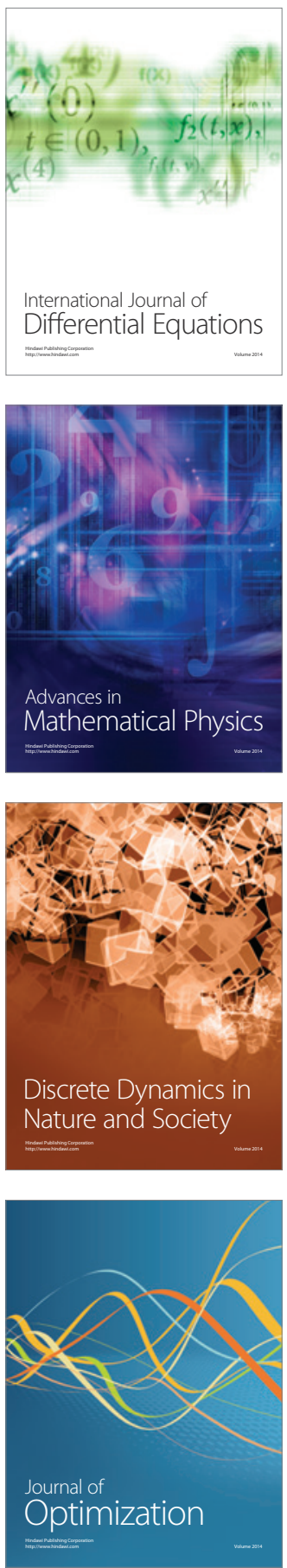